BORON CLUSTERS

\section{Taken for a spin}

Angew. Chem. Int. Ed. http://doi.org/fz5gq7 (2012)

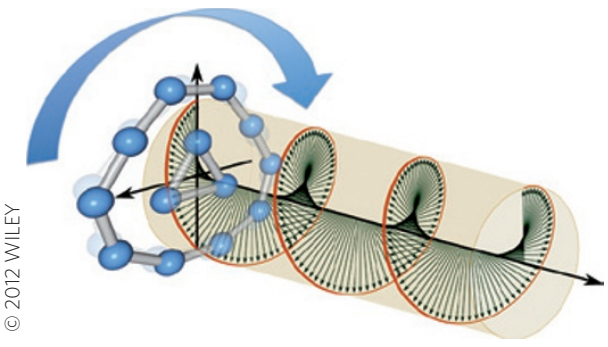

A key design feature of both macroscopic and nanoscopic motors is energy efficiency. On the nanoscale, molecular motors can convert light, chemical or electrical energy into directed motion through the excitation of electronic states in the molecule. However, if the motors could operate only on the potential energy surface of the electronic ground state they would be more energy efficient. Jin Zhang, Anastassia Alexandrova and colleagues from the University of California Los Angeles and Utah State University have now shown that boron clusters composed of 13 atoms could be used as such a device.

The planar $\mathrm{B}_{13}{ }^{+}$clusters are composed of an inner triangular ring of atoms surrounded by an outer ring of ten atoms. Previously it was shown that the outer ring of atoms can rotate with respect to the inner ring due to delocalized bonding, but such thermally driven motion is bidirectional. With the aid of theoretical calculations, Zhang,
Alexandrova and colleagues demonstrate that unidirectional rotation can be achieved by using a circularly polarized infrared laser as an energy source. In particular, the researchers examine the energy profile of two elementary rotational moves of the outer ring - one clockwise, the other counterclockwise and find that without an electric field both directions are equally possible. However, when a constant electric field is applied, the counterclockwise rotation is strongly favoured: the transition-state barrier for counterclockwise rotation almost completely disappears, whereas the barrier for clockwise rotation increases significantly.

With circularly polarized infrared radiation near $3 \mathrm{THz}$, the rotating frequency of the outer ring would be approximately $250 \mathrm{GHz}$ and the US team suggest that the electric field strength used in their simulations could be achieved experimentally with ultraintense lasers.

\section{ELECTRODES}

With gold in mind

Nano Lett. 12, 3391-3398 (2012)

Making the electrodes in neural prosthetic devices small and flexible reduces the inflammation and scarring they cause. However, most materials are unable to deliver a sufficiently high charge per area, and have too high an interfacial impedance when they are fashioned into sufficiently small electrodes. The high electron mobility and surface area of carbon nanotubes
NANOPARTICLE FACTORIES Health hazards investigated

Two types of nanoparticles can enter the environment: unintentionally produced incidental nanoparticles from pollution or combustion products, and intentionally produced engineered nanomaterials such as carbon nanotubes. Epidemiological studies have shown a link between the number of ambient ultrafine particles and respiratory illnesses. Moreover, several cell culture and animal studies have shown that when inhaled, engineered particles with diameters of less than $100 \mathrm{~nm}$ can enter the lungs, circulate in the blood and cause harmful effects such as inflammation, cancer and heart problems. Now, researchers in Taiwan have shown that few adverse health effects are detected in factory workers who handle engineered nanoparticles.

Saou-Hsing Liou of the Division of Environmental Health and Occupational Medicine at the National Health Research Institute in Taiwan and co-workers from various institutes in Taiwan used a questionnaire to collect data on exposure status and demographics, and measured the urine and blood samples of the factory workers for biomarkers that could indicate health effects. Fourteen factories with a total of 227 exposed workers and 137 unexposed workers participated, and the risk level of each worker was categorized according to the nanotool risk-level matrix, where risk level is scored based on the severity of the toxicity of the nanomaterial and the probability of exposure. Exposed workers had lower levels of antioxidant enzymes and increased cardiovascular markers than unexposed workers. However, no differences were seen in lung function or markers for lung inflammation, oxidative stress or genotoxicity amongst all workers, suggesting little adverse response.

recommend them as electrodes, but their performance at small dimensions and their long-term biocompatibility remain an issue. Nicholas Kotov and colleagues at the University of Michigan have now demonstrated that gold nanoparticle electrodes may be a better choice for neural prosthetics.

The researchers constructed two small electrodes, one from carbon nanotubes and one from gold nanoparticles. Layerby-layer assembly was used to ensure that each electrode had the same thickness (around $100 \mathrm{~nm}$ ). The impedance of the gold nanoparticle electrode was measured to be three times lower, and the charge storage capacity (a measure of available charge per area) ten times higher, than the carbon nanotube electrode. Moreover, a large body of clinical data supports the long-term biocompatibility of gold. The gold electrode performance benefited from a high surface area, and from good connectivity among nanoparticles.

\section{TWO-DIMENSIONAL MATERIALS Mix and match}

Nano Lett. 12, 3518-3525 (2012)

The successful isolation of graphene flakes from graphite has prompted researchers to seek other two-dimensional materials that can be obtained by exfoliation from stacked bulk solids. Recent examples include hexagonal boron nitride (h-BN), $\mathrm{MoS}_{2}$ and $\mathrm{WS}_{2}$. Pulickel Ajayan, Angel Rubio, Yansheng Yin and colleagues have now shown that it is possible to prepare a stacked film made of alternating nanosheets of h-BN and graphene.

The researchers - who are based at institutes in the US, Spain, China and India - first obtain graphene and h-BN flakes separately by ordinary exfoliation, and then simply combine the two materials under sonication to obtain free-standing hybrid films. Spectroscopic investigations show that the hexagonal arrangement of both the carbon atoms, and the boron and nitrogen atoms is generally maintained. Interplane covalent bonds are also possible, due to the presence of some dangling bonds, and may contribute to the stability of the film.

Theoretical calculations hint at original optoelectronic properties stemming from the interaction at the interface between materials with dissimilar electronic properties. For example, h-BN (an insulator) could absorb ultraviolet light and directly inject charges into the graphene (a conductor) layer.

Written by Ai Lin Chun, Alberto Moscatelli, Michael Segal and Owain Vaughan. 